\title{
Hyperchaotic Oscillation in the Deformed Rikitake Two-Disc Dynamo System Induced by Memory Effect
}

\author{
Yanling Wang, ${ }^{1}$ Tengfei Lei, ${ }^{1}$ Xin Zhang $\mathbb{D},{ }^{2}$ Chunbiao Li $\mathbb{D},{ }^{1,2}$ and Sajad Jafari $\mathbb{C}^{3}$ \\ ${ }^{1}$ Collaborative Innovation Center of Memristive Computing Application (CICMCA), Qilu Institute of Technology, \\ Jinan 250200, China \\ ${ }^{2}$ Jiangsu Collaborative Innovation Center of Atmospheric Environment and Equipment Technology, \\ Nanjing University of Information Science \& Technology, Nanjing 210044, China \\ ${ }^{3}$ Biomedical Engineering Faculty, Amirkabir University of Technology, 424 Hafez Ave, Tehran 15875-4413, Iran
}

Correspondence should be addressed to Chunbiao Li; goontry@126.com

Received 29 January 2020; Revised 14 March 2020; Accepted 13 April 2020; Published 6 May 2020

Guest Editor: Chun-Lai Li

Copyright (C) 2020 Yanling Wang et al. This is an open access article distributed under the Creative Commons Attribution License, which permits unrestricted use, distribution, and reproduction in any medium, provided the original work is properly cited.

The fundamental dynamics of the deformed Rikitake two-disc dynamo system is explored in this paper. Memory effect on the dynamical behavior of the generator system is studied by introducing a quadratic flux-controlled memristor. Hyperchaotic oscillation in the deformed Rikitake two-disk coupled generator is therefore firstly found. Lyapunov exponents, bifurcation diagram, and phase portraits prove the abundant dynamic behavior consistently.

\section{Introduction}

Memristor is a passive element with nonlinearity and nonvolatility. According to the completeness of basic circuit components, in 1971, Chua first predicted the existence of the fourth circuit component, which describes the relationship between charge and flux [1]. In 2008, Hewlett-Packard Laboratory successfully fabricated the nanoscale memristor based on metal and metal oxides [2], which aroused great interest in the scientific and technological community. Recently, great progress has been made in the research of memristors, and the application of memristoralso has become a hot focus. Because of the small size, low power consumption, nonlinearity, and non-volatility, the memristor can be applied in many areas such as nonlinear chaotic circuits [3-6], electronic engineering [7-9], artificial intelligence [10-12], and neural networks [13-15].

It is considered that the geomagnetic field is associated with the conductive outer core, which has been proved by some dynamical models. In 1958, T. Rikitake firstly proposed a Two-Disc Dynamo System (RTDDS) and observed this physical law [16]. RTDDS is a simple model to demonstrate the polarity reversal of the earth's magnetic field, in which the current from each disc excites the coil of the other [17]. In fact, it is a chaotic system [18] and exhibits abundant dynamical behavior [19-21].

Recently, much attention has been paid to the RTDDS. A new attractor synthesis algorithm was applied to model the attractors in the Rikitake system [22]. By applying synchronization technique based on control theory, an active controller was designed for the synchronization of two identical RTDDS [19]. A method to stabilize asymptotically the nontrivial Lyapunov stable states of Rikitake two-disk dynamo dynamics was given in [23]. It was proven that the two non-hyperbolic equilibrium points of the Rikitake system are all stable for all positive parameters [24]. A simple realization of the symmetric Rikitake system was given in [25]. A reduced-order projective synchronization system was designed for the Rikitake system without any equilibria or with two non-hyperbolic equilibrium points in [26]. In [27], a 4-D hyperchaotic Rikitake dynamo system without any equilibria was proposed. In [28], a 5-D hyperchaotic Rikitake dynamo system with three positive Lyapunov exponents was proposed, which has a hidden attractor without any equilibrium point. From aforementioned references, the 
hyperchaotic system of RTDDS was constructed by artificial numerical methods directly through feedback and other means.

With the continuous development of industrial automation, intelligent motors with control and learning capabilities have also been realized. In this paper, a deformed RTDDS is constructed by introducing an extra flux-controlled memristor. The motor can be controlled to avoid the chaotic region through the combination of memristors and memristor control parameters. After the memristor is added, the bifurcation point of the system is changed, and the original behavior state of the RTDDS is also changed. Therefore, the research in this paper provides an important idea for the control of the double-disk motor. This system is proven with abundant dynamical behaviors, including a line of equilibria and hyperchaos [29-33]. In Section 2, the brief introduction of the flux-controlled memristor is given, thereafter a new lossy RTDDS is given, and the deformed RTDDS is constructed by adding one extra flux-controlled memristor. Rich dynamics of the presented system are analyzed in Section 3. In Section 4, the analog circuit of the new memristive hyperchaotic system is implemented based on Multisim simulation. Some conclusions are finally drawn in Section 5.

\section{Modeling of the Deformed Two- Disc Generator}

2.1. Memristor Model. According to the relationship between voltage $v$ and current of a flux-controlled memristor $[1,34,35]$, choose a cubic nonlinearity to describe the $q$ function [36-38], and then, $W(\varphi)$ is

$$
\left\{\begin{array}{l}
i=W(\varphi) y, \\
W(\varphi)=\alpha+3 \beta \varphi^{2}, \\
\dot{\varphi}=y
\end{array}\right.
$$

where $\alpha$ and $\beta$ are two positive constants. Figure 1 shows the voltage-current relationship of the memristor with $\alpha=2$ and $\beta=0.1$ under the frequency $f=1 \mathrm{~Hz}$. The hysteresis curve agrees the inherent characteristics of the memristor.

In the following, the above memristor is applied to study the memory effect of the deformed RTDDS.

2.2. Description of the Deformed RTDDS. In 1958, Rikitake first proposed a two-disc generator, in which the dimensionless equations are $[16,18]$

$$
\left\{\begin{array}{l}
\dot{x}_{1}=-\mu x_{1}+x_{2} x_{3}, \\
\dot{x}_{2}=-\mu x_{2}+x_{1}\left[x_{3}-\mu\left(\sigma^{2}-\sigma^{-2}\right)\right], \\
\dot{x}_{3}=1-x_{1} x_{2}
\end{array}\right.
$$

where $x_{1}$ and $x_{2}$ are dimensionless currents, $x_{3}$ is the angular velocity of the two discs, $\mu$ and $\sigma$ are adjustable parameters.

In the chaotic double-disk generator, considering the wear rate of the double-disk generator, the model of the deformed double-disk coupled generator is constructed under electromechanical coupling. The new deformed RTDDS is proposed as

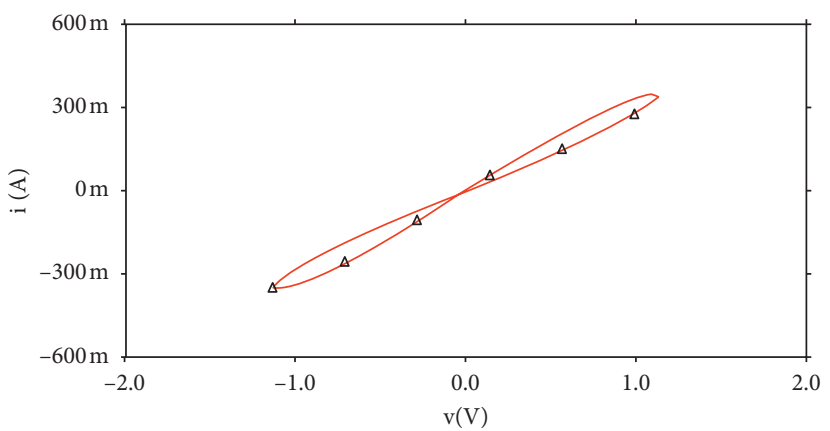

FIgURE 1: Pinched hysteresis loop of the memristor with $\alpha=2$ and $\beta=0.1(f=1 \mathrm{~Hz})$.

$$
\left\{\begin{array}{l}
\dot{x}=-a x+y(z+c), \\
\dot{y}=-b y+x(z-c) \\
\dot{z}=d z-x y
\end{array}\right.
$$

where $a$ and $b$ are the ratio of resistance to self-inductance of the two loops, which represent the dissipative performance of the generator, and are closely related to the working conditions of the generator. Here, $c$ is the difference in angular velocity between two rotors of the coupled generators, $d$ is the wear parameter, $x$ and $y$ represent the current across the two loops, and $z$ is the angular velocity of the double discs. System (3) with $a=2, b=3, c=5$, and $d=0.75$ has a chaotic solution with Lyapunov exponents: $L E_{1}=0.2825, L E_{2}=0$, and $L E_{3}=-4.4179$ under the initial condition (0.1, 0.1,0.1), as shown in Figure 2. System (3) shows rotational symmetry since it is recovered by the transformation $(x, y, z) \longrightarrow(-x,-y, z)$.

2.3. Memristive Deformed RTDDS. In RTDDS, assume that the loop current of the first disc depends on the change of the current of the other disc, and the memory effect can be indicated by the memristor. A flux-controlled memristor is applied in RTDDS with $\alpha=2$ and $\beta=0.1$, and the memristive deformed RTDDS is

$$
\left\{\begin{array}{l}
\dot{x}=-a x+y z+k W(\varphi) y, \\
\dot{y}=-b y+x(z-c) \\
\dot{z}=\mathrm{d} z-x y \\
\dot{\varphi}=y
\end{array}\right.
$$

where $a, b, c$, and $d$ are positive parameters, and $k$ is a positive parameter representing the strength of the memristor. System (4) with $a=2, b=3, c=5, d=0.75$, and $k=1$ has a hyperchaotic attractor with Lyapunov exponents $L E_{1}=0.3784, L E_{2}=0.0218, L E_{3}=0.0000$, and $L E_{4}=-4.6503$, two of which are positive indicating hyperchaos, as shown in Figure 3. The Kaplan-York dimension is $D_{\mathrm{KY}}=3.0861$. Poincaré map is a line representing chaos if the surface represents hyperchaos, and the Poincaré map of the system is shown in Figure 4; in this system, Poincaré map is surface, so it is hyperchaos. The invariance of system (4) under the transformation $(x, y, z, \varphi) \longrightarrow(-x,-y, z,-\varphi)$ shows the 


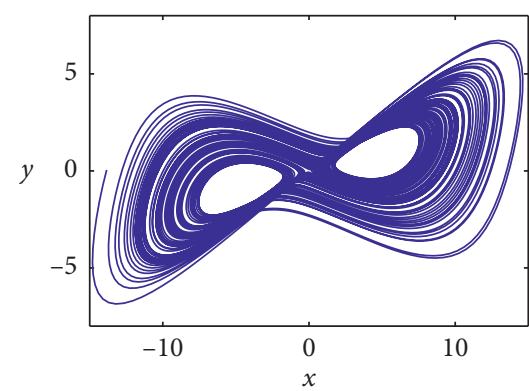

(a)

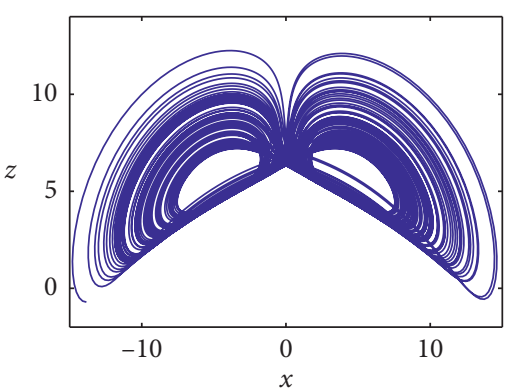

(b)

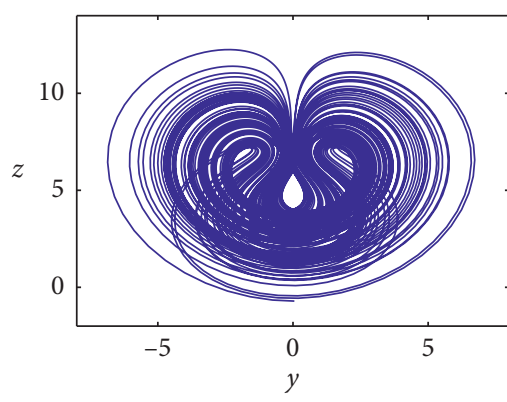

(c)

Figure 2: Phase portraits of system (3) with $a=2, b=3, c=5$, and $d=0.75$ : (a) $x$-y plane, (b) $x$ - $z$ plane, and (c) $y$ - $z$ plane.

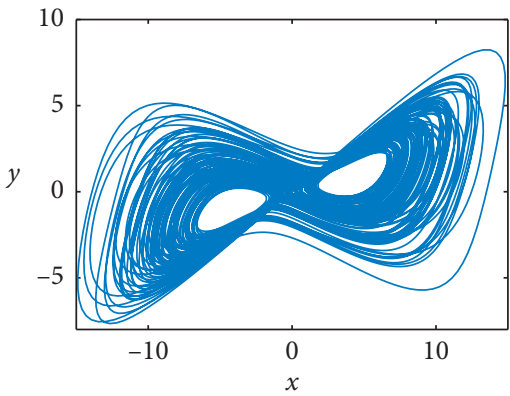

(a)

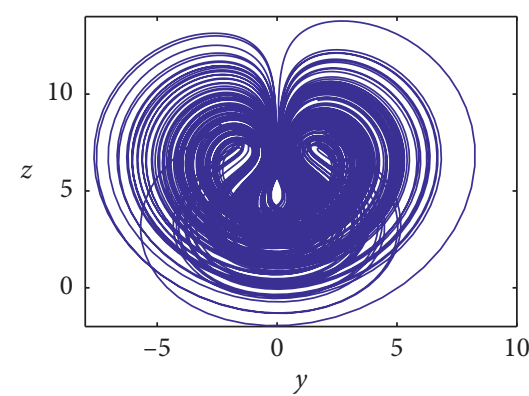

(b)

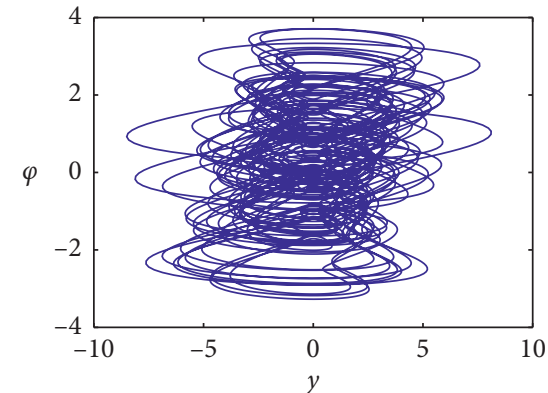

(c)

Figure 3: Phase portraits of system (4) with $a=2, b=3, c=5, d=0.75$, and $k=1$ : (a) $x-y$ plane, (b) $y-z$ plane, and (c) $y$ - $\varphi$ plane.

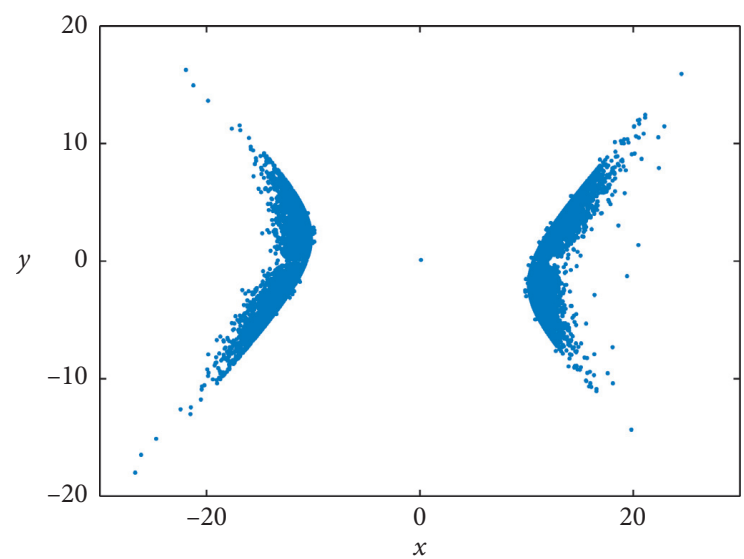

(a)

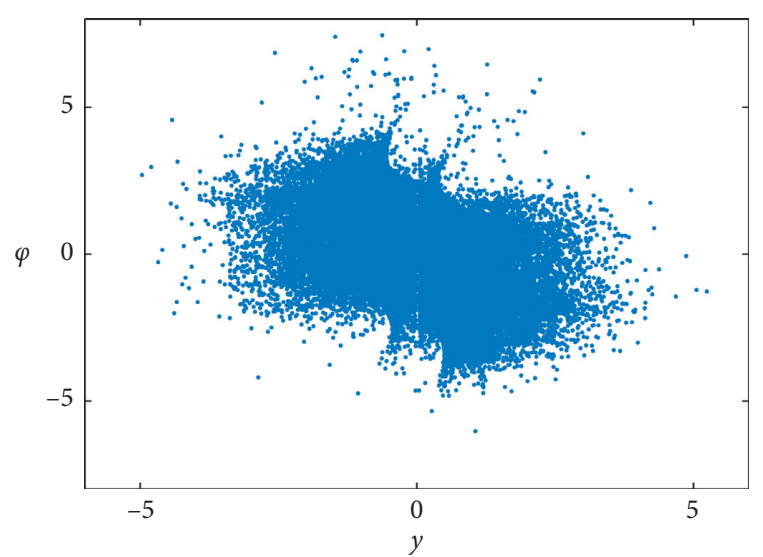

(b)

Figure 4: Poincaré map of system (4) with $a=2, b=3, c=5, d=0.75$, and $k=1$ : (a) $x$-y plane; (b) $y$ - $\varphi$ plane.

rotational symmetric structure. Therefore, newly introduced memristor transforms the system to be a hyperchaotic one.

\section{Dynamical Behaviours of the Proposed Chaotic System}

3.1. Line of Equilibria and Stability Analysis. The equilibrium points of system (4) can be derived by solving the following equations:

$$
\left\{\begin{array}{l}
-a x+y z+k W(\varphi) y=0 \\
-b y+x(z-c)=0 \\
\mathrm{~d} z-x y=0 \\
y=0
\end{array}\right.
$$

System (5) has a line of equilibria $[0,0,0, \varphi]$, where $\varphi$ is a real variable. By linearizing system (5) at the equilibria, the Jacobian matrix can be obtained: 


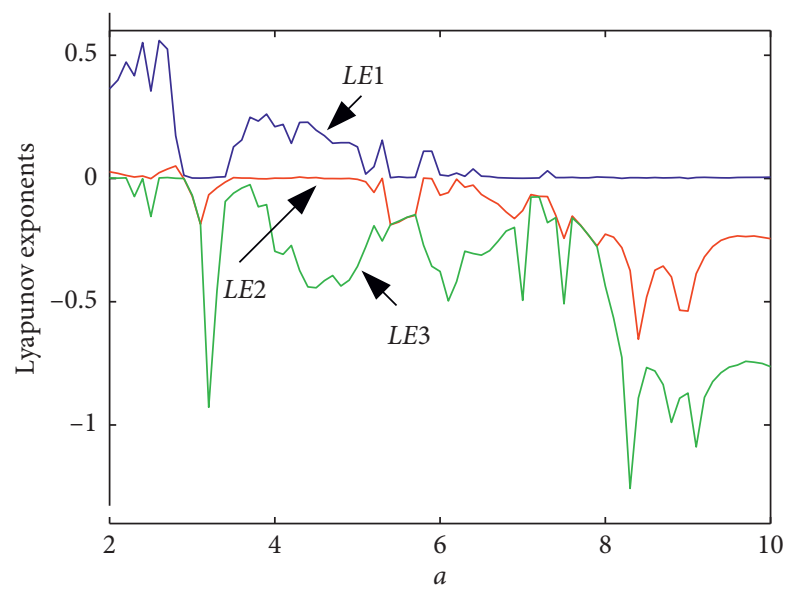

(a)

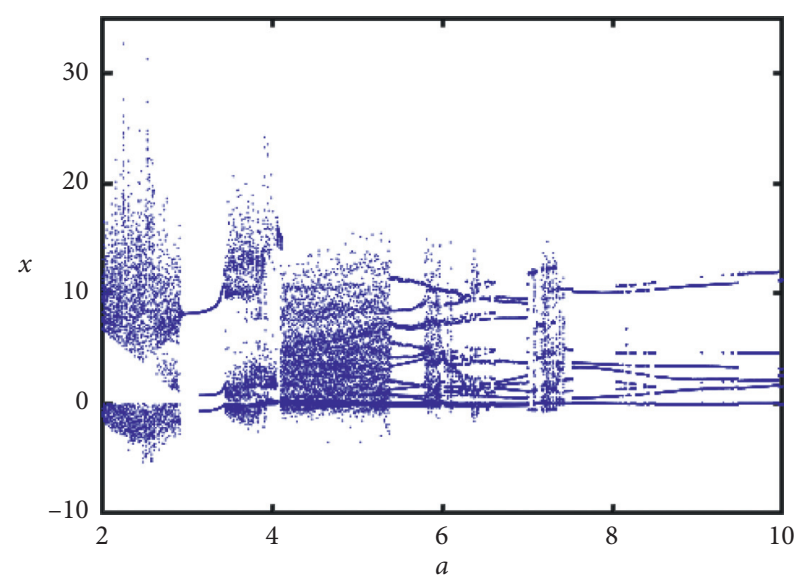

(b)

FiguRE 5: Lyapunov exponent spectra and bifurcation of system (4) with $b=3, c=5, d=0.75$, and $k=1$, and $a$ varies in [2, 10]: (a) Lyapunov exponent spectra: $L E_{1}$ is blue, $L E_{2}$ is red, and $L E_{3}$ is green; (b) bifurcation diagram.

$$
J o=\left[\begin{array}{cccc}
-a & k W(\varphi) & 0 & 0 \\
-c & -b & 0 & 0 \\
0 & 0 & d & 0 \\
0 & 1 & 0 & 0
\end{array}\right] .
$$

According to equation (6), the characteristic equation can be obtained as

$$
\lambda(\lambda-d)\left[\lambda^{2}+(a+b) \lambda+a b+c k W(\varphi)\right]=0 .
$$

So, the eigenvalues are

$$
\begin{aligned}
\lambda_{1} & =0, \\
\lambda_{2} & =d, \\
\lambda_{3,4} & =\frac{-(a+b) \pm \sqrt{(a-b)^{2}-4 c k W(\varphi)}}{2} .
\end{aligned}
$$

Therefore, the stability of the line equilibria is associated with the values of $a, b, c, k$, and $W(\varphi)$. From $\lambda_{2}$ and $\lambda_{3,4}$, it can be seen that when $a, b$, and $d$ are positive, the line equilibria point is unstable, command $\Delta_{k}=(a-b)^{2}-$ $4 c k W(\varphi)$, where if $\Delta_{\mathrm{k}}>0$, and the line equilibria are unstable nodes or else if $\Delta_{\mathrm{k}}<0$, the line equilibria are unstable saddle foci. Specifically, when $a=2, b=3, c=5, d=0.75$, and $k=1$, the line equilibria are unstable saddle focus.

3.2. Dynamical Analysis. The dynamic behavior of system (4) will be further investigated with Lyapunov exponent spectra and bifurcation diagram.

When $b=3, c=5, d=0.75, k=1$, and $a$ varies in $[2,10]$, set the initial condition $I C=(0.1,0.1,0.1,0.1)$, step size of $a$ is 0.01 , time step is $0.01 \mathrm{~s}$, and running time is $300 \mathrm{~s}$; the corresponding Lyapunov exponent spectra and bifurcation diagram are obtained as shown in Figure 5. As shown in Figure 5(a), when $a \in[0,2.58)$, the largest Lyapunov exponent is positive, so system (4) is chaotic; when $a \in[2.58,2.9)$, two of the Lyapunov exponents are positive. Thus, system (4) is hyperchaotic; thereafter, system (4) enters into the periodic mode at $a=2.9$. It remains periodic when $a \in[2.9,3.42)$. When $a \in[3.42,5.33) \cup[5.81,6.4) \cup[7.24,7.6)$, it stays in chaos; system (4) drops in the periodic state occasionally when $a \in[5.33,5.81) \cup[6.4,7.24) \cup[7.6,10)$. Bifurcation diagram shown in Figure 5(b) agrees with the Lyapunov exponents. Specific periodic oscillations are shown Figure 6, and the detail information of Lyapunov exponents is shown in Table 1. We noticed that system (4) sometimes provides a symmetric oscillation and sometimes gives a symmetric pair of limit cycles.

The memristor bridges the loop current of two disks. Different phase portraits can be obtained under different initial values, as shown in Figure 7. A symmetric oscillation or a symmetric pair of limit cycles is found simultaneously.

Let $a=2, b=3, d=0.75$, and $k=1$, while $c$ varies from 0 to 10 , and the initial condition $\mathrm{IC}=(0.1,0.1,0.1,0.1)$, the Lyapunov exponent spectra, and the corresponding bifurcation diagram are shown in Figure 8. As shown in Figure 8, when $c \in[0,1.9) \cup[4.6,8) \cup[8.24,10)$, system (4) is chaotic; when $c \in[2.7,4.6)$, system (4) is hyperchaotic; and when $c \in$ $[1.9,2.64) \cup[8,8.42)$, system (4) is periodic. Let $a=2, b=3$, $c=5$, and $d=0.75$, while $k$ varies in $[0,5]$, set the initial condition $I C=(0.1,0.1,0.1,0.1)$, and the Lyapunov exponent spectra and the corresponding bifurcation diagram are shown in Figure 9, showing system (4) stays in chaos robustly. Usually, a memristive system shows multistability induced by the memory effect, however when $a=2, b=3$, $c=5, d=0.75$, and $k=1$, let the initial condition $I C=(0.1,0.1$, $0.1, \varphi), \varphi$ varies from -5 to 5 , the Lyapunov exponent spectra and the corresponding bifurcation diagram are shown in Figure 10, showing system (4) stays in chaotic orbit.

\section{Circuit Simulation Based on Multisim}

In order to further observe the specific hyperchaotic oscillation behavior induced from the memory effect, a circuit 


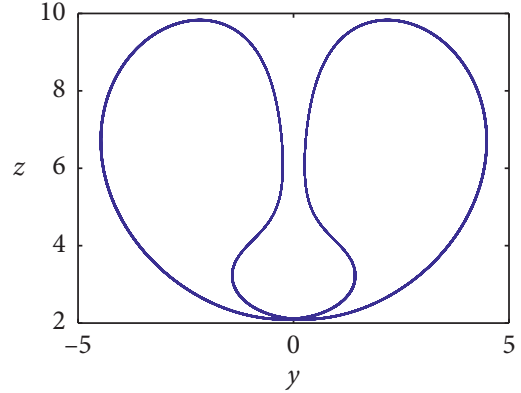

(a)

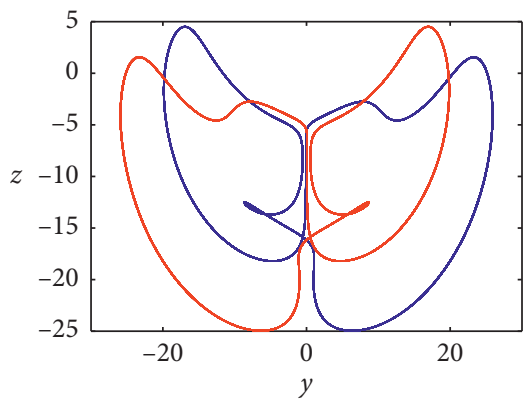

(d)

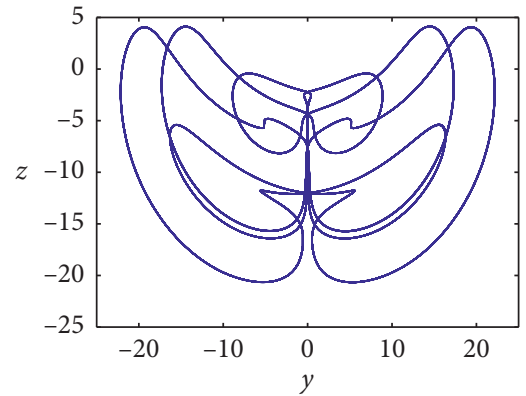

(b)

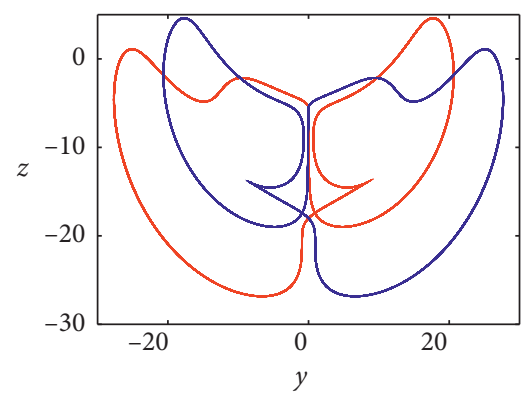

(e)

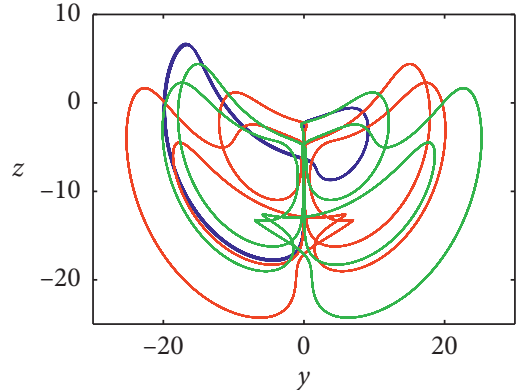

(c)

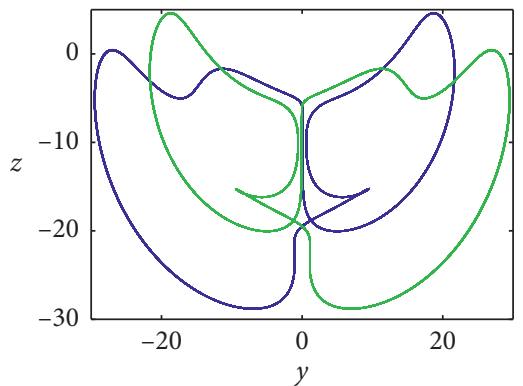

(f)

Figure 6: Various periodic oscillations in system (4) with $b=2, c=5, d=0.75$, and $k=1$ and (a) $a=3.2$, (b) $a=5.8$, (c) $a=7$, (d) $a=8.5$, (e) $a=9$, and (f) $a=9.8, I C=(0.1,0.1,0.1,0.1)$ is blue, $(-01,0.1,0.1,0.1)$ is red, and $(0.1,0.1,-0.1,0.1)$ is green.

TABLE 1: Periodic oscillations in system (4) under different parameters of $a$.

\begin{tabular}{lccc}
\hline Cases & Parameter $a$ & Lyapunov exponents & Solution type of system (4) \\
\hline A & $a=3.2$ & $0.0002-0.0665-0.9399-4.4432$ & Symmetric attractor \\
B & $a=5.8$ & $0.12140 .0000-0.3017-7.8699$ & Symmetric attractor \\
C & $a=7$ & $0.0005-0.1337-0.5036-8.6132$ & Asymmetric attractor \\
D & $a=8.5$ & $0.0000-0.4863-0.7792-9.4844$ & Asymmetric attractor \\
E & $a=9$ & $0.0014-0.5440-0.8858-9.8216$ & Asymmetric attractor \\
F & $a=9.8$ & $0.0006-0.2361-0.7574-11.0571$ & Asymmetric attractor \\
\hline
\end{tabular}

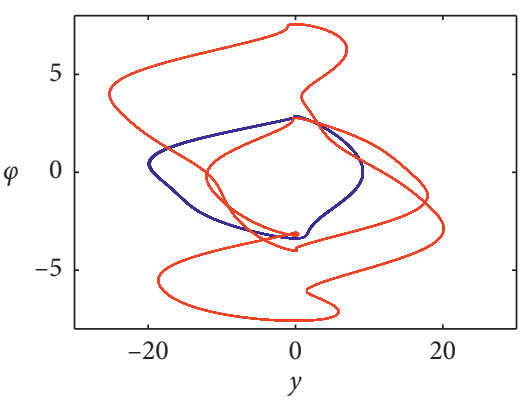

(a)

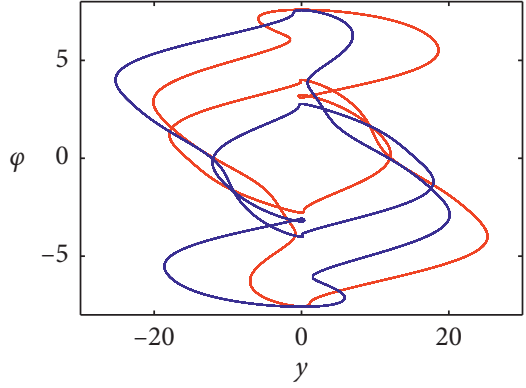

(b)

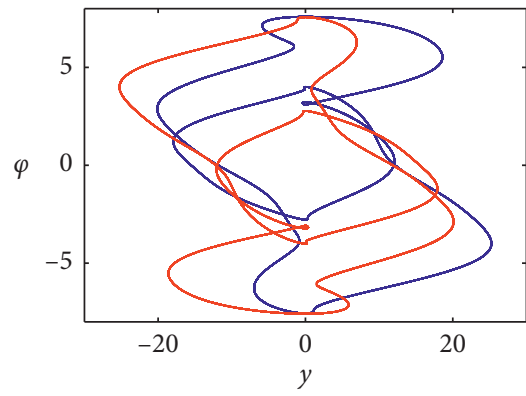

(c)

FIGURE 7: Various periodic oscillations in system (4) with $a=7, b=3, c=5, d=0.75$, and $k=1$ : (a) $I C=(0.1,0.1,0.1,0.1)$ is blue, $(0.1,-0.1,0.1$, $0.1)$ is red, (b) $I C=(0.1,5,0.1,0.1)$ is blue, $(0.1,-5,0.1,0.1)$ is red, and (c) $I C=(0.1,10,0.1,0.1)$ is blue, $(0.1,-10,0.1,0.1)$ is red.

simulation is realized based on Multisim software [39]. In the circuit design, resistors, capacitors, operational amplifiers (OPA404AG), analog multipliers, and other elements are applied. The supply voltages for OPA404AG operational amplifiers (with saturated voltages $V_{\text {sat }} \approx \pm 13.5 \mathrm{~V}$ ) are $\pm 15 \mathrm{~V}$. In fact, the variable $z$ is beyond the normal operating range of the device. Therefore, here, the variables are transformed by proportional compression, that is, $v_{x}=10 v_{x 1}, v_{y}=10 v_{y 1}$, $v_{z}=10 v_{z 1}$, and $v_{\varphi}=10 v_{\varphi 1}$, where $v_{x 1}, v_{y 1}, v_{z 1}$, and $v_{\varphi 1}$ are the voltages on the integral capacitor, respectively. By time rescaling of system (4), the equations can be obtained as follows: 


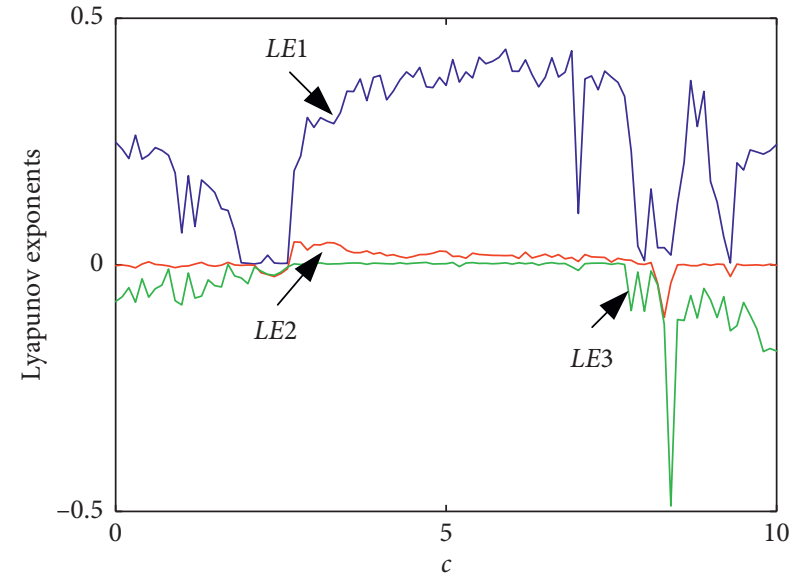

(a)

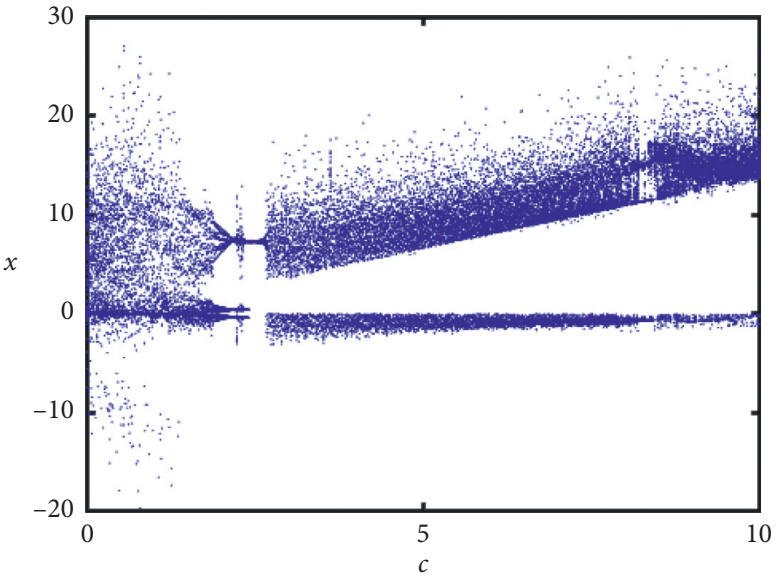

(b)

FIGURE 8: Lyapunov exponent spectra and bifurcation diagram of system (4) with $a=2, b=3, d=0.75$, and $k=1$, while $c$ varies in [1, 10]: (a) Lyapunov exponent spectra: $L E_{1}$ is blue, $L E_{2}$ is red, and $L E_{3}$ is green; (b) bifurcation diagram.

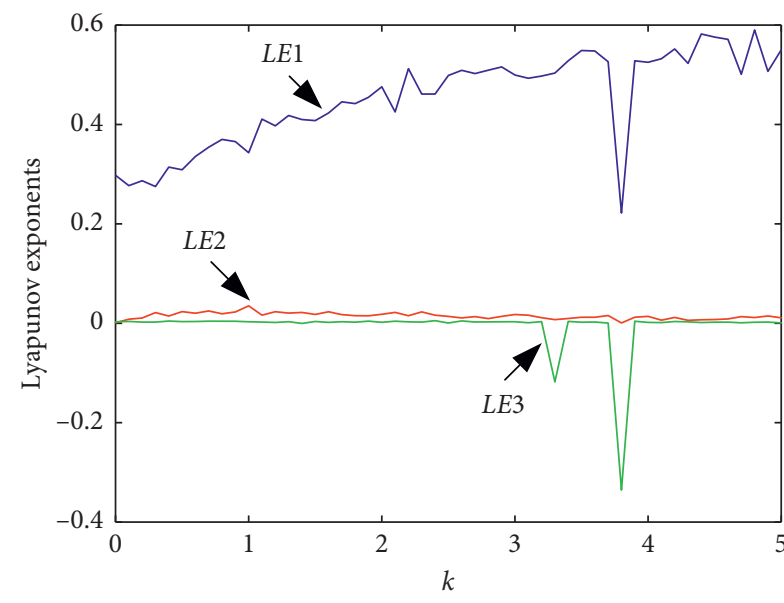

(a)

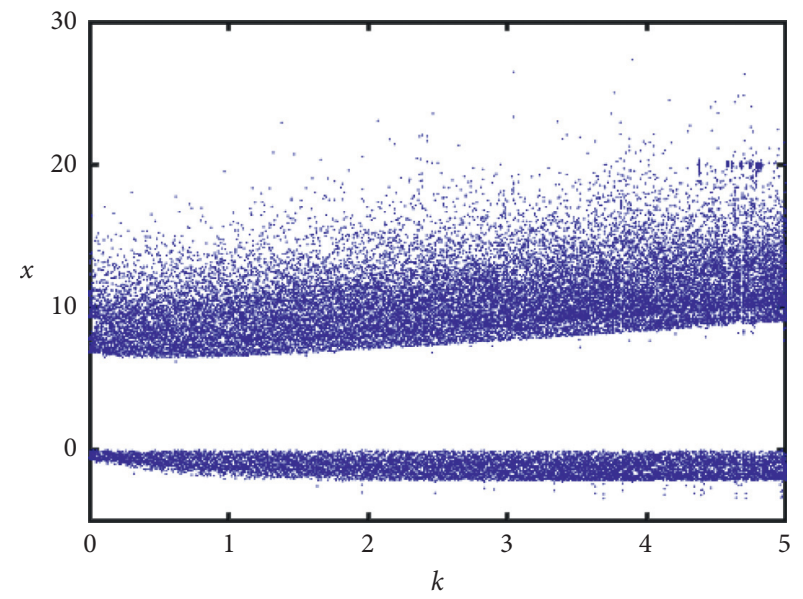

(b)

FIGURE 9: Lyapunov exponent spectra and bifurcation diagram of system (4) with $a=2, b=3, c=5$, and $d=0.75$, while $k$ varies in [0,5]: (a) Lyapunov exponent spectra: $L E_{1}$ is blue, $L E_{2}$ is red, and $L E_{3}$ is green; (b) bifurcation diagram.

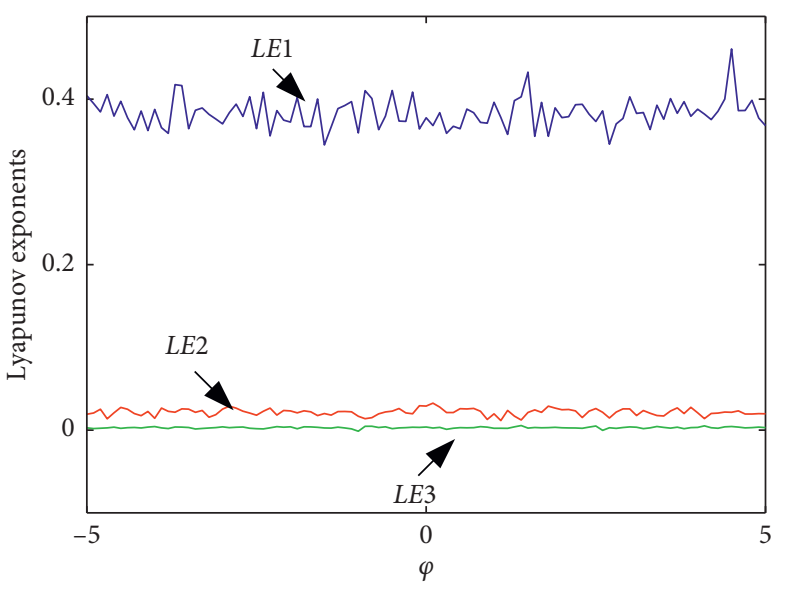

(a)

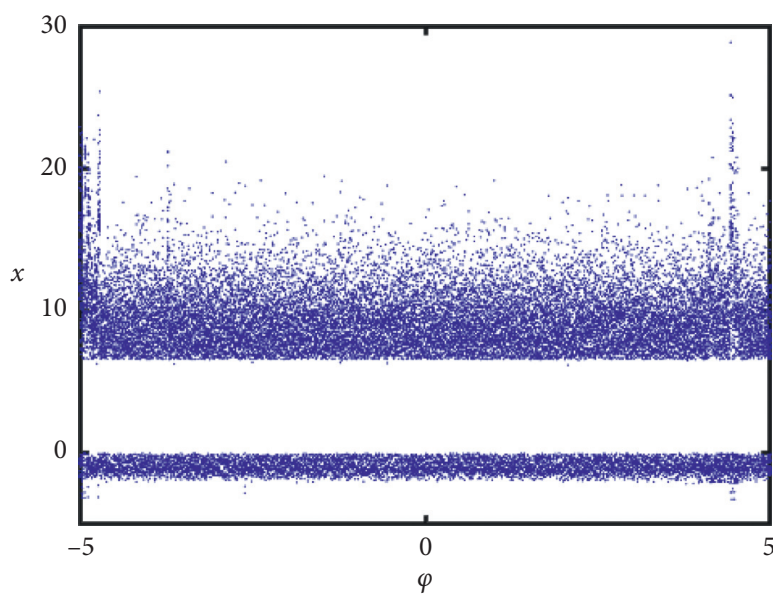

(b)

FIGURE 10: Lyapunov exponent spectra and bifurcation diagram of system (4) with $a=2, b=3, c=5, d=0.75$, and $k=1$, while $\varphi$ varies in [-5, 5]: (a) Lyapunov exponent spectra: $L E_{1}$ is blue, $L E_{2}$ is red, and $L E_{3}$ is green; (b) bifurcation diagram. 

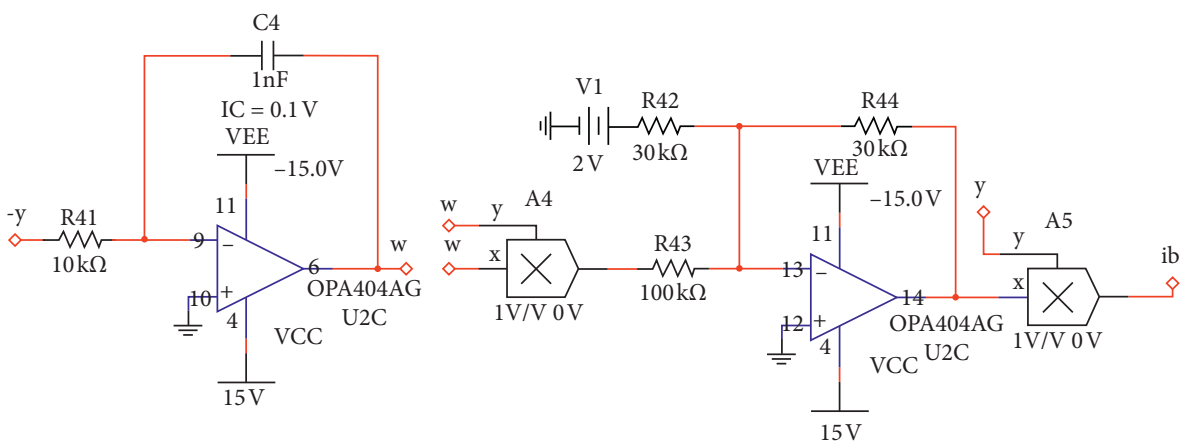

(a)
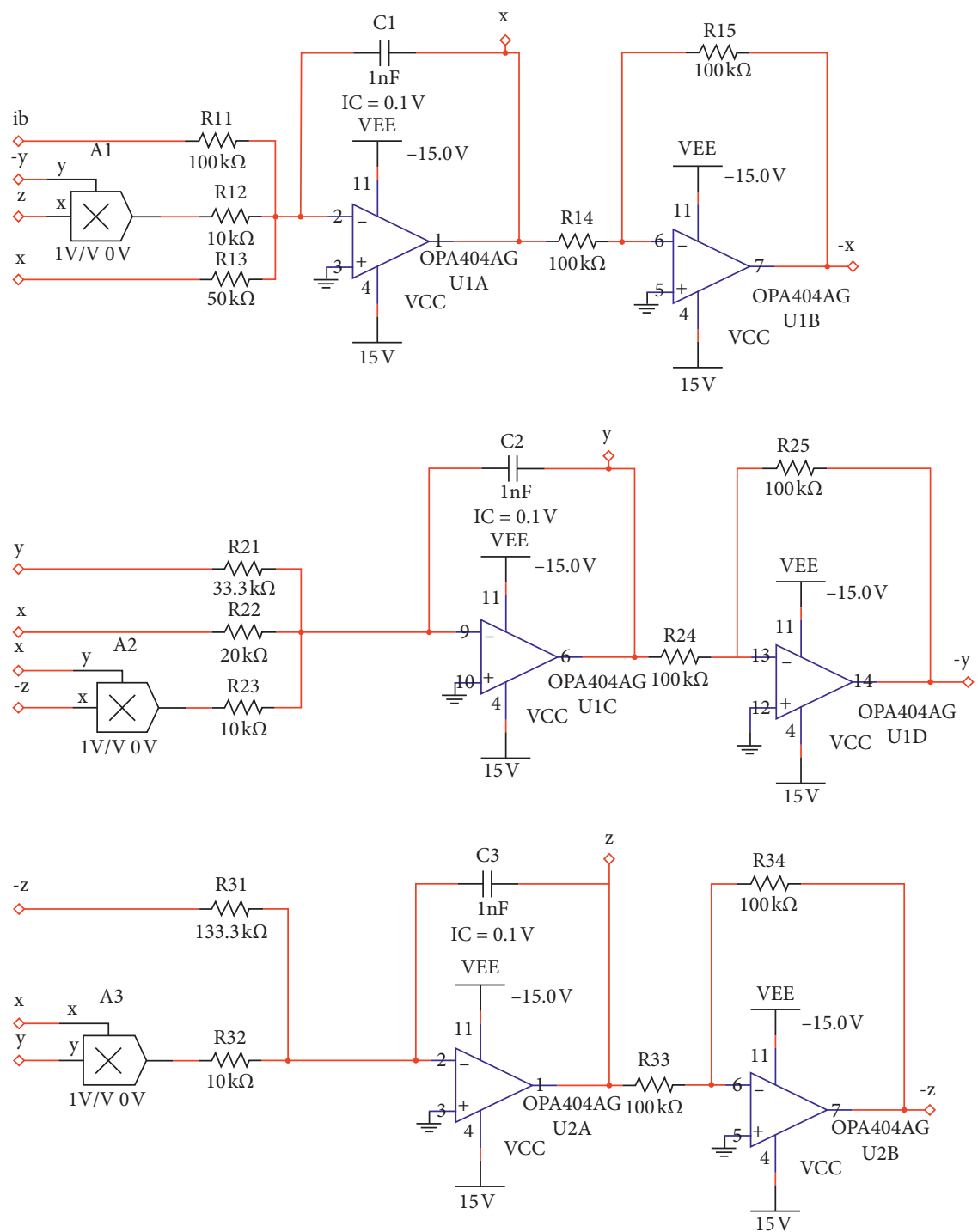

(b)

Figure 11: The analog circuit of the deformed RTDDS. 


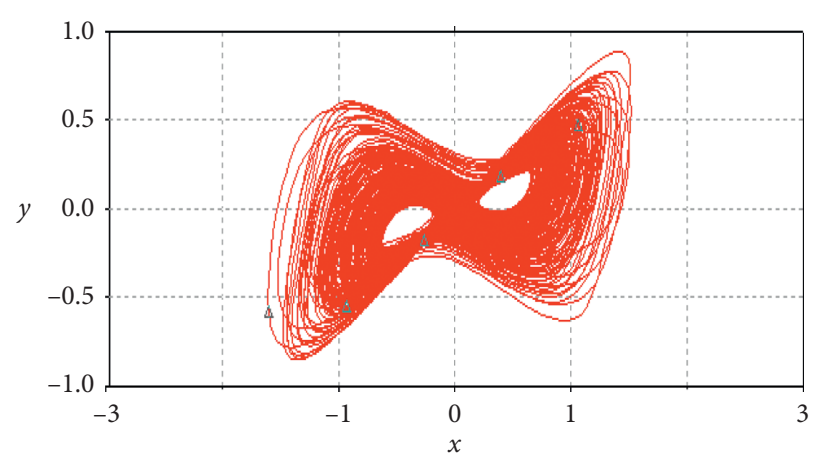

(a)

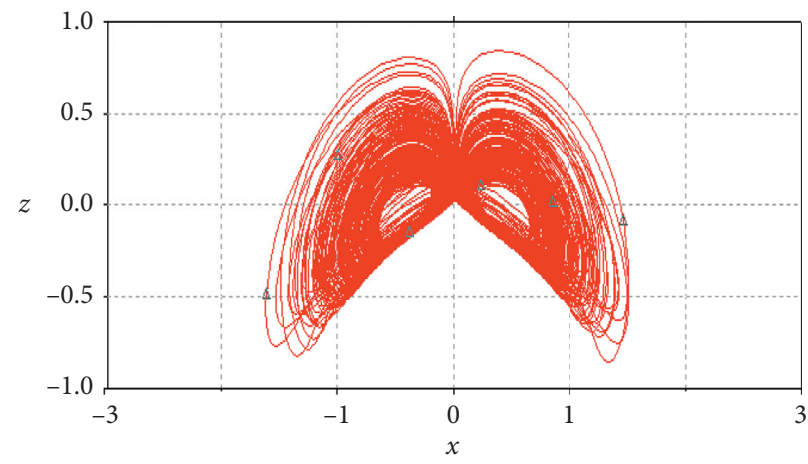

(c)

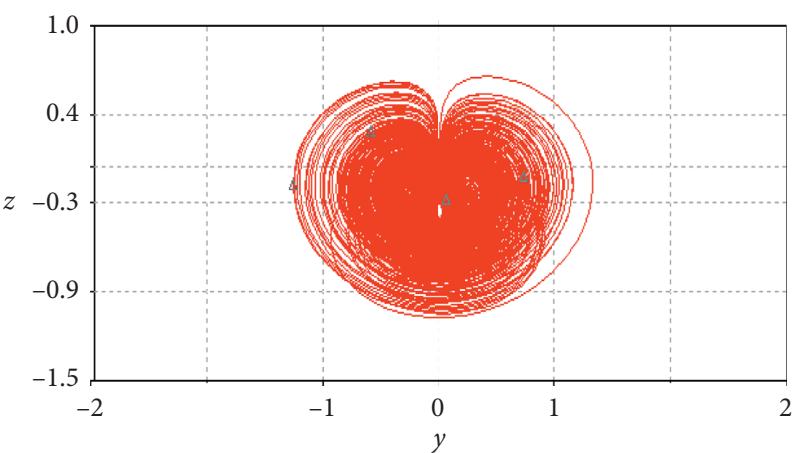

(b)

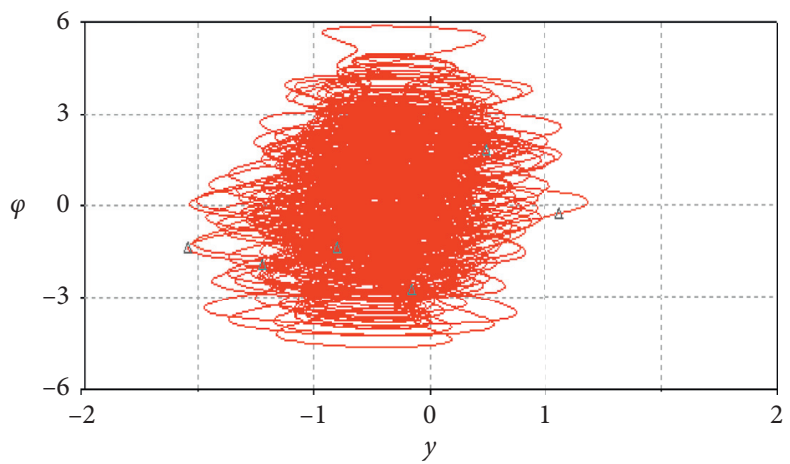

(d)

FiguRE 12: Simulation results obtained from Multisim software: (a) attractor on the $x-y$ plane, (b) attractor on the $y$ - $z$ plane, (c) attractor on the $x-z$ plane, and $(\mathrm{d})$ attractor on the $y-\varphi$ plane.

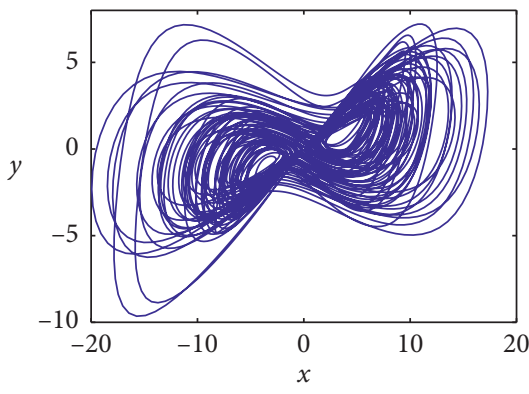

(a)

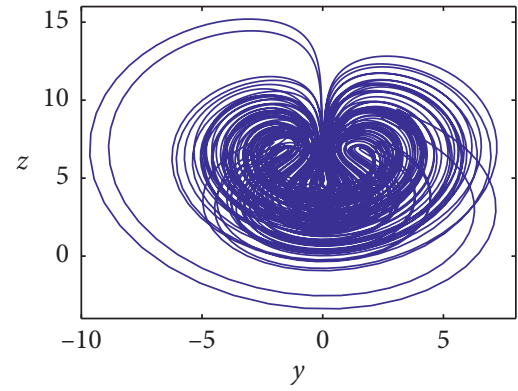

(b)

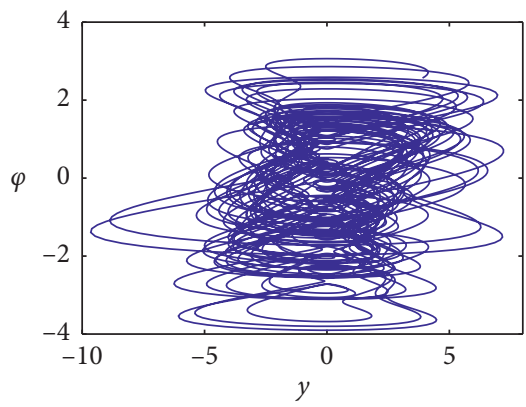

(c)

FIGURe 13: Phase portraits of system (4) with memristor $W(\varphi)=-m+n|\varphi|, m=2, n=5$, and $a=2, b=3, c=5, d=0.75$, and $k=1$ : (a) $x-y$ plane, (b) $y-z$ plane, and (c) $y-\varphi$ plane.

$$
\left\{\begin{array}{l}
C_{1} \dot{v}_{x}=-\frac{1}{R_{13}} v_{x}+\frac{1}{R_{12}} v_{y} v_{z}+\frac{1}{R_{11}} W(\varphi) v_{y}, \\
C_{2} \dot{v}_{y}=-\frac{1}{R_{21}} v_{y}+\frac{1}{R_{22}} v_{x} v_{z}-\frac{1}{R_{23}} v_{x}, \\
C_{3} \dot{v}_{z}=\frac{1}{R_{31}} v_{z}-\frac{1}{R_{32}} v_{x} v_{y}, \\
C_{4} \dot{v}_{\varphi}=\frac{1}{R_{41}} v_{y} .
\end{array}\right.
$$

The corresponding analog circuit is shown in Figure 11. The circuit in Figure 11(a) represents the quadratic nonlinear flux-controlled memristor, which consists of an integration circuit and a proportional circuit. Resistor R41, operational amplifier U2C, and capacitor C4 constitute an integral circuit, which integrates the voltage $v$ across the memristor giving the magnetic flux through the memristor.

In Figure 11, the capacitances and resistances are $C_{1}=C_{2}=C_{3}=C_{4}=1 \mathrm{nF}, R_{12}=R_{23}=R_{32}=R_{41}=10 \mathrm{k} \Omega, R_{13}=$ $50 \mathrm{k} \Omega, \quad R_{21}=33.3 \mathrm{k} \Omega, \quad R_{22}=20 \mathrm{k} \Omega, \quad R_{31}=133.3 \mathrm{k} \Omega$, $R_{42}=R_{44}=30 \mathrm{k} \Omega$, and $R_{14}=R_{15}=R_{24}=R_{25}=R_{33}=R_{34}=$ $R_{43}=R_{11}=100 \mathrm{k} \Omega$. Figure 12 gives the phase trajectories shown in the oscilloscopes. 
In order to verify the memory effect in RTDDS, a new flux-controlled memristor $W(\varphi)=-m+n|\varphi|$, with $m=2$ and $n=5$, is introduced. System (4) has a hyperchaotic attractor with Lyapunov exponents $L E_{1}=0.3225$, $L E_{2}=0.0554, L E_{3}=0.0000$, and $L E_{4}=-4.6309$ when $a=2$, $b=3, c=5, d=0.75$, and $k=1$, two of which are positive indicating hyperchaos, as shown in Figure 13.

\section{Conclusions and Discussion}

The deformed Rikitake two-disc dynamo system possesses rich dynamics including chaos, hyperchaos, and different periodic oscillations. A memristive deformed RTDDS was constructed for observing the memory effect. Consequently, an analog circuit based on the flux-controlled memristor was designed for further verification. Circuit simulation agrees with the theoretical analysis and numerical simulation. By the memristor model built the control circuit, through memristor and matching parameters of the memristor, the RTDDS can be controlled to avoid the chaotic region and realize the smooth operation. This research provides a meaningful reference for motor design and control.

\section{Data Availability}

The data used to support the findings of this study are available from the corresponding author upon request.

\section{Conflicts of Interest}

The authors declare that they have no conflicts of interest.

\section{Acknowledgments}

This work was supported financially by the National Nature Science Foundation of China (Grant nos. 61871230, 51974045, and 61971228), the Natural Science Foundation of Jiangsu Province (Grant no. BK20181410), the Startup Foundation for Introducing Talent of NUIST (Grant no. 2016205), and a project funded by the Priority Academic Program Development of Jiangsu Higher Education Institutions, Major Scientifific and Technological Innovation Projects of Shandong Province (Grant no. 2019JZZY010111), the Natural Science Foundation of Shandong Province (Grant no. ZR2017PA008), the Key Research and Development Plan of Shandong Province (Grant no. 2019GGX104092), and Science and Technology Plan Projects of Universities of Shandong Province (Grant no. J18KA381).

\section{References}

[1] L. Chua, "Memristor-The missing circuit element," IEEE Transactions on Circuit Theory, vol. 18, no. 5, pp. 507-519, 1971.

[2] D. B. Stukov, G. S. Snider, D. R. Stewart et al., "The missing memristor found," Nature, vol. 453, pp. 80-83, 2008.

[3] B. C. Bao, Z. Liu, and J. P. Xu, "Steady periodic memristor oscillator with transient chaotic behaviours," Electronics Letters, vol. 46, no. 3, p. 228, 2010.
[4] C. Li, W. Joo-Chen Thio, H. Ho-Ching Iu, and T. Lu, "A memristive chaotic oscillator with increasing amplitude and frequency," IEEE Access, vol. 6, pp. 12945-12950, 2018.

[5] J. Wang, G. Iu, and T. Fernando, IEEE Transactions on Circuits System, vol. 28, 2018.

[6] G. Ella, International Journal of Bifurcation and Chaos in Applied Sciences and Engineering, vol. 29, pp. 246-250, 2014.

[7] H. Chang, Z. Wang, Y. Li, and G. Chen, Semiconductor Science and Technology, vol. 65, pp. 104004-104014, 2017.

[8] L. Xie, H. A. D. Nguyen, M. Taouil, S. Hamdioui, and K. Bertels, "A mapping methodology of boolean logic circuits on memristor crossbar," IEEE Transactions on ComputerAided Design of Integrated Circuits and Systems, vol. 37, no. 2, pp. 311-323, 2018.

[9] X. Wang, Q. Wu, Q. Chen, and Z. Zeng, "A novel design for memristor-based multiplexer via NOT-material implication," IEEE Transactions on Computer-Aided Design of Integrated Circuits and Systems, vol. 37, no. 7, pp. 1436-1444, 2018.

[10] M. Vaynshteyn and A. Lanis, "Applications of electrochemical elements in systems of artificial intelligence," Natural Science, vol. 11, pp. 45-51, 2013.

[11] S. Liu, Y. Wang, M. Fardad, and P. K. Varshney, "A memristor-based optimization framework for artificial intelligence applications," IEEE Circuits and Systems Magazine, vol. 18, no. 1, pp. 29-44, 2018.

[12] J. H. Yoon, Z. Wang, K. M. Kim et al., "An artificial nociceptor based on a diffusive memristor," Nature Communications, vol. 9, p. 417, 2018.

[13] I. E. Ebong and P. Mazumder, "CMOS and memristor-based neural network design for position detection," Proceedings of the IEEE, vol. 100, no. 6, pp. 2050-2060, 2012.

[14] B. Bao, H. Qian, J. Wang et al., "Numerical analyses and experimental validations of coexisting multiple attractors in Hopfield neural network," Nonlinear Dynamics, vol. 90, no. 4, pp. 2359-2369, 2017.

[15] M. Di Marco, M. Forti, and L. Pancioni, "New conditions for global asymptotic stability of memristor neural networks," IEEE Transactions on Neural Networks and Learning Systems, vol. 29, no. 5, pp. 1822-1834, 2018.

[16] T. Rikitake, "Oscillations of a system of disk dynamos," Mathematical Proceedings of the Cambridge Philosophical Society, vol. 54, 1958.

[17] A. E. Cook and P. H. Roberts, "The Rikitake two-disc dynamo system," Mathematical Proceedings of the Cambridge Philosophical Society, vol. 68, no. 2, pp. 547-569, 1970.

[18] I. Keisuke, "Chaos in the Rikitake two-disc dynamo system," Earth and Planetary Science Letters, vol. 51, pp. 451-456, 1980.

[19] U. E. Vincent, "Synchronization of Rikitake chaotic attractor using active control," Physics Letters A, vol. 343, no. 1-3, pp. 133-138, 2005.

[20] M. T. Răzvan and A. Gîrban, Nonlinear Analysis: Real World Applications, vol. 11, pp. 2888-2895, ScienceDirect, Edinburgh, London, 2009.

[21] J. Llibre and M. Messias, "Global dynamics of the Rikitake system," Physica D: Nonlinear Phenomena, vol. 238, no. 3, pp. 241-252, 2009.

[22] M.-F. Danca and S. Codreanu, "Modeling numerically the Rikitake's attractors by parameter switching," Journal of the Franklin Institute, vol. 349, no. 3, pp. 861-878, 2012.

[23] R. A. Tudoran, Nonlinear Analysis: Real World Applications, vol. 12, pp. 2505-2510, Elsevier, Oxford, UK, 2011.

[24] D. C. Braga, F. S. Dias, and L. F. Mello, "On the stability of the equilibria of the Rikitake system," Physics Letters A, vol. 374, pp. 4316-4320, 2010. 
[25] C. Lăzureanu and T. Bînzar, "On the symmetries of a Rikitake type system," Comptes Rendus-Mathématique, vol. 350, pp. 529-533, 2012.

[26] F. Yu and W. Q. Pan, "Hidden attractors without equilibrium and adaptive reduced-order function projective synchronization from hyperchaotic Rikitake system," Pramana, vol. 88, pp. 1-6, 2017.

[27] S. Vaidyanathan, C. K. Volos, and V.-T. Pham, "Analysis, adaptive control and adaptive synchronization of a nine-term novel 3-D chaotic system with four quadratic nonlinearities and its circuit simulation," Journal of Engineering Science and Technology Review, vol. 8, pp. 232-244, 2015.

[28] S. Vaidyanathan, V.-T. Pham, and C. K. Volos, "A 5-D hyperchaotic Rikitake dynamo system with hidden attractors," The European Physical Journal Special Topics, vol. 224, no. 8, pp. 1575-1592, 2015.

[29] S. Jafari and J. C. Sprott, "Simple chaotic flows with a line equilibrium," Chaos, Solitons \& Fractals, vol. 57, pp. 79-84, 2013.

[30] K. Sun, X. Wang, and J. C. Sprott, "Bifurcations and chaos in fractional-order simplified lorenz system," International Journal of Bifurcation and Chaos, vol. 20, no. 4, pp. 1209-1219, 2010.

[31] G. Zhang, P. Qian, and Z. Su, "Evolution of fractional-order chaotic economic systems based on non-degenerate equilibrium points," Chaos, Solitons \& Fractals, vol. 128, pp. 219-228, 2019.

[32] C. B. Li, J. C. Sprott, W. Thio, and H. Q. Zhu, "A new piecewise linear hyperchaotic circuit," IEEE Transaction on Circuits and System II Express Briefs, vol. 61, p. 977C981, 2014.

[33] B. Bao, P. Jiang, $\mathrm{H}$. Wu, and F. Hu, "Complex transient dynamics in periodically forced memristive Chua's circuit," Nonlinear Dynamics, vol. 79, no. 4, pp. 2333-2343, 2015.

[34] L. O. Chua and S. M. Sung Mo Kang, "Memristive devices and systems," Proceedings of the IEEE, vol. 64, no. 2, pp. 209-223, 1976.

[35] M. D. Ventra, Y. V. Pershin, and L. O. Chua, "Circuit elements with memory: Memristors, memcapacitors, and meminductors," Proceedings of the IEEE, vol. 97, pp. 1717-1724, 2009.

[36] B. Muthuswamy, "Implementing memristor based chaotic circuits," International Journal of Bifurcation and Chaos, vol. 20, no. 5, pp. 1335-1350, 2010.

[37] H. H. C. Iu, D. S. Yu, A. L. Fitch, V. Sreeram, and H. Chen, "Controlling chaos in a memristor based circuit using a twinT notch filter," IEEE Transactions on Circuits and Systems I: Regular Papers, vol. 58, no. 6, pp. 1337-1344, 2011.

[38] B. C. Bao, J. P. Xu, G. H. Zhou, Z. H. Ma, and L. Zou, "Chaotic memristive circuit: equivalent circuit realization and dynamical analysis," Chinese Physics B, vol. 20, 2011.

[39] Y. Sui, Y. He, W. Yu, and Y. Li, "Design and circuit implementation of a five-dimensional hyperchaotic system with linear parameter," International Journal of Circuit Theory and Applications, vol. 46, no. 8, pp. 1503-1515, 2018. 